potential for refining our approach to making judgements about causal relationships in public health.

\section{OP116 ASSOCIATION OF EPILEPSY WITH DEMENTIA: A RETROSPECTIVE NATIONWIDE DATA LINKAGE COHORT STUDY}

${ }^{1} \mathrm{C}$ Schnier*, ${ }^{2} \mathrm{GK}$ Mbizvo, ${ }^{1,2} \mathrm{~T}$ Wilkinson, ${ }^{2} \mathrm{SE}$ Duncan, ${ }^{2} \mathrm{RFM}$ Chin. ${ }^{1}$ Usher Institute of Population Health Sciences and Informatics, The University of Edinburgh, Edinburgh, UK; ${ }^{2}$ Muir Maxwell Epilepsy Centre, The University of Edinburgh, Edinburgh, UK

\subsection{6/jech-2019-SSMabstracts. 115}

Background People with epilepsy (PWE) are reported to have higher risk of dementia. However, the magnitude of this association, whether it varies according to dementia subtypes and whether there are factors that modify the association is uncertain. We investigated the apparent association in a large population-based retrospective cohort study using routinely-collected linked health data from hospitalisation, mortality records and primary care consultations.

Methods We used linked health data from the Secure Anonymised Information Linkage (SAIL) databank to follow-up Welsh residents for whom linked primary care data were available from their 60th birthday to estimate dementia rate and associated risk factors. Disease (dementia), exposure (epilepsy) and covariates (medication, smoking, stroke and diabetes) were classified using previously validated code lists. We studied rate of dementia and dementia subtypes in people with and without epilepsy using (stratified) KaplanMeier plots and flexible parametric proportional hazard analyses.

The study population comprised 563,808 people of whom $19,807(4 \%)$ had indications of epilepsy in the linked health data. 13,454 (68\%) of PWE and 49,439 (9\%) of people without epilepsy had at least one record for a prescription of an antiepileptic drug (AED). Compared to people without epilepsy, PWE had lower survival (median survival to age 79 years compared to 84$)$, higher smoking risks $(74 \%$ compared to $66 \%)$ and higher stroke risks (20\% compared to $7 \%$ ) before or during follow-up.

Results Between ages 60 and 70 years, 6\% of PWE and 1\% of people without epilepsy had a diagnosis of dementia; corresponding figures between ages 60 and 80 years were 23\% and $8 \%$. The difference in dementia rate between those with and without epilepsy was larger for vascular dementia than for Alzheimer's disease. The increased rate for PWE was modified by a history of stroke, smoking and, to a lesser effect, diabetes. PWE who were first diagnosed before age 25 years had a lower dementia rate than those diagnosed later in life. Compared to PWE not exposed, those exposed to sodium valproate were at higher risk of dementia (crude HR: 1.7; 95\% CI: 1.5-1.9) while those exposed to a group of enzymeinducing AED were at similar risk (crude HR: 1.1, 95\% CI: 1.0-1.3).

Conclusion At least some of the increased risk of dementia in PWE can be attributed to increased vascular risk factors in PWE causing vascular dementia. Given the widespread use of sodium valproate in PWE, the association of the drug with higher dementia risk is concerning.
Rapid Fire Programme

Friday 6 September

Chronic Disease

\section{RF01 EDUCATIONAL OUTCOMES AMONG CHILDREN WITH TYPE 1 DIABETES: WHOLE-OF-POPULATION STUDY}

${ }^{1,2} \mathrm{M}$ Begum* ${ }^{*},{ }^{1,2} \mathrm{CR}$ Chittleborough, 1,2 RM Pilkington, 1,2 M Mittinty, 1,2,3 JW Lynch,

${ }^{1,2,4} \mathrm{M}$ Penno, ${ }^{1,2} \mathrm{LG}$ Smithers. 'School of Public Health, The University of Adelaide, Adelaide, South Australia; ${ }^{2}$ Robinson Research Institute, The University of Adelaide, Adelaide, South Australia; ${ }^{3}$ Population Health Sciences, University of Bristol, UK; ${ }^{4}$ School of Medicine, University of Adelaide, Adelaide, South Australia

\subsection{6/jech-2019-SSMabstracts.116}

Background Evidence about the impact of type 1 diabetes (T1D) on educational outcomes is mixed. Despite advances in clinical care and intensive insulin treatment regimens, achieving optimum metabolic control is a challenge in pediatric populations with T1D. Poor metabolic control leading to hyperglycemia or hypoglycemia can potentially have implications for children's educational outcomes. In the last decade, there has been substantial improvement in T1D management, therefore the objective of this study was to estimate to what extent T1D is linked to children's educational outcomes.

Methods This whole-of-population study $(n=61,445)$ used deidentified, administrative linked data from the South Australian Early Childhood Data Project (births 1999-2013). This study examined the impact of $\mathrm{T} 1 \mathrm{D}$ on reading, writing, spelling, grammar and numeracy scores of children in year 5 (age 10 years), assessed by the National Assessment Program-Literacy and Numeracy (NAPLAN) in 2008-2015. Children with T1D were identified from hospitalization data (2001-2014) using ICD-10-AM diagnosis codes (E10, E101-E109).

The effect of T1D on the five NAPLAN domains (continuous variables) was estimated by augmented inverse probability treatment weighting (AIPW). AIPW includes; 1) creation of weights and, 2) using those weights in the outcome regression in a way such that the final estimates of the treatment effect is unbiased, even if the weights regression or the outcome regression is incorrect. We explored two associations between T1D and educational outcomes; 1) T1D versus non-T1D, 2) time since diagnosis ( $\leq 2$ years, $3-10$ years) versus non-T1D. Additionally, to address the problem of missing data we used multiple imputation.

Results Among 61,445 children born in South Australia and who had undertaken NAPLAN assessments, 162 had been diagnosed with T1D. There was no difference in the mean reading, writing, spelling, and grammar and numeracy scores of children with and without T1D. For example, the crude mean reading score was 482.8 with a standard deviation of 78.9 , and the average treatment effect was 6.84 (95\% CI $6.25,19.92)$, which reflects a negligible difference in the mean reading scores of children with and without T1D. There was also no difference in educational outcome between children who were recently diagnosed (exposed to T1D for $\leq 2$ years), or those who were exposed to T1D for 3-10 years at the time of NAPLAN assessment, compared with non-T1D.

Conclusion This whole-of-population study demonstrated that children with T1D are not performing poorly on literacy or 